\title{
Layer-Oriented single- and dual-field-of-view performance for OWL
}

\author{
Enrico Fedrigo ${ }^{* a}$, Enrico Marchetti ${ }^{\mathrm{a}}$, Carmelo Arcidiacono ${ }^{\mathrm{b}}$ Emiliano Diolaiti ${ }^{\mathrm{c}}$, \\ ${ }^{a}$ European Southern Observatory; ${ }^{b}$ Università di Firenze - Dipartimento di Astronomia e Scienza \\ dello Spazio; ${ }^{\mathrm{D}}$ Dipartimento di Astronomia - Università di Padova
}

\begin{abstract}
Layer-Oriented MCAO is a promising technique that can scale up easily compared to similar competing techniques. The Multiple-Field-of-View Layer-Oriented makes a further step employing more guide stars to sample the turbulence with the net effect of increasing the sky coverage, thereby enabling MCAO systems based only natural guide stars.

However even a simple estimation of Layer-Oriented M-FoV MCAO performance for Extremely Large Telescopes, in particular for OWL, has not been attempted yet. The main problem is the enormous amount of computing power (processor and memory) required to properly carry out an accurate simulation for ELTs.

In this work we perform a numerical simulation using some shortcuts to simplify the problem and achieve a first cut of what the performance of Layer-Oriented M-FoV MCAO could be for OWL. We take a mixed analytical-numerical approach in order to significantly reduce the amount of computing power required while maintaining a good level of accuracy.
\end{abstract}

Keywords: AO, MCAO, Multiple FoV, Layer-Oriented, 100m Telescopes, OWL

\section{INTRODUCTION}

Multi-Conjugate Adaptive Optics (MCAO) has been first proposed by Beckers [1,2] to extend the corrected Field of View (FoV) typically limited in the classical Adaptive Optics (AO) systems [3]. In a MCAO systems several Deformable Mirrors (DM) are conjugated to different altitudes above the telescope and the atmospheric correction is done in a three-dimensional way. Each DM corrects the part of the atmosphere which it is conjugated to, even if the vertical discretization is somewhat rough. In order to reconstruct the vertical distribution of the atmospheric turbulence, different approaches have been proposed. Tallon and Foy [16] introduced the concept of tomography to disentangle numerically the turbulence at fixed altitudes, using independent measurements obtained from a number of Guide Stars (GS) through classical Wave Front Sensors (WFS), like Shack-Hartmann or Curvature sensors. Later Ragazzoni, Marchetti and Rigaut [8] introduced a more effective concept of modal tomography were the tomography approach is performed in modal way.

The validity of the method has also been proven on the sky [10] although in a preliminary form. At the same time the novel concept of layer-oriented has been introduced [9,11] where many GS are simultaneously sensed with a single WFS with several detectors conjugated at the DM conjugation altitudes. The signal from each detector drives its corresponding DM allowing efficient closed-loop operations. Layer-oriented approach is extremely effective in terms of Signal-to-Noise Ratio optimization of the sensing process because it is possible to tune both the temporal and the spatial sampling for the temporal $\left(\tau_{0}\right)$ and spatial frequencies $\left(r_{0}\right)$ characteristic of the layer which the detector is conjugated to. Moreover the co-addition of the light of the GS in a single plane allows lowering the requirements in GS brightness and, in this way, to increase the sky coverage when Natural GS (NGS) are considered.

In the layer-oriented approach any kind of pupil plane WFS could be suitable but a quite promising one is the Pyramid WFS [7]. Furthermore an extension of the layer-oriented approach, called Multiple FoV [12,13], allow to significantly increase the Sky Coverage using a larger FoV for the ground conjugated detector to collect light from more stars. Extremely Large Telescopes [5] can strongly benefit from the layer-oriented approach and moreover the Multiple FoV concept gives really competitive Sky Coverage with respect to Laser GS based systems.

In this paper we present preliminary results of simulations for the Single and Multiple Field of View, (S-FoV and MFoV respectively) here with only two fields and thus called Dual Field of View (D-FoV). It should be noted here that

* enrico.fedrigo@eso.org; phone +49.89.32006324; fax +49.89.3202362; http://www.eso.org; European Southern Observatory; Karl-Schwarzschild-Str. 2; D-85748 Garching bei München; Germany 
the simulations we are going to describe were made with several simplifications in order to make it achievable and indepth verification of the results is still needed.

In section 2 we describe the parameters of the various modules of the simulation, while in section 3 we describe the software architecture of the simulation tool. We verify the results of the simulations in section 4 , while section 5 is devoted to a comparison between OWL and the VLT Multi-Conjugate Adaptive Optics Demonstrator (MAD) to tune the simulation parameters in preparation of section 6 , where we present our results with the OWL configuration.

\section{SIMULATION PARAMETERS}

\subsection{Model of the atmosphere}

We simulated the atmosphere using multiple phase screens. The number of phase screens and their size is one of the most important parameters that affects the computing power required to complete each run of the simulation. We then decided to simplify as much as possible. Our shortcuts are as follows:

- We limited the number of phase screens to 4 . We took a $\mathrm{C}_{\mathrm{n}}{ }^{2}$ profile and we computed the best approximation of that profile with only 4 layers, computing their altitude and strength.

- We limited the size of the phase screens to a grid of 2048 by 2048 pixels. Since each pixel of the phase screen is a float this results in a $16 \mathrm{MB}$ memory occupation per layer, 64MB total just to load the atmosphere model.

- The wind can only flow in two directions: up-down (or reverse) and left-right (or reverse), at any speed

- Phase screens contain $\mathrm{L}_{0}$ and they are symmetrical

- The phase screens are rotated locally without interpolation and no Fresnel propagation is implemented.

We generated the atmosphere using a Kolmogorov power spectrum modified with the addition of an outer scale of $22 \mathrm{~m}$. The second fundamental parameter of the simulation software is the size in pixels of the pupil of the telescope: this is the main parameter since each operation on the atmosphere will be performed cutting pupils of that size from the various layers and combining them together in various ways. We decided to set the size of the OWL pupil to 1000 pixels, thus the pixel scale becomes $0.1 \mathrm{~m}$. We also decided to work in good seeing conditions: 0.60 " at $550 \mathrm{~nm}$.

\begin{tabular}{|c|c|c|c|c|}
\hline Phase screen size & \multicolumn{4}{|c|}{$2048 \times 2048$} \\
\hline Pupil & \multicolumn{4}{|c|}{$100 \mathrm{~m}$} \\
\hline Pixels across the pupil & \multicolumn{4}{|c|}{$1000 \times 1000$} \\
\hline Pixel scale & \multicolumn{4}{|l|}{0.1} \\
\hline 10Km metapupil 2' FoV (diameter) & \multicolumn{4}{|c|}{$105.82 \mathrm{~m}$} \\
\hline Pixel across 2’ FoV metapupil @ 10Km & \multicolumn{4}{|l|}{1058} \\
\hline 10Km metapupil 6' FoV (diameter) & \multicolumn{4}{|c|}{$117.45 \mathrm{~m}$} \\
\hline $\mathrm{r}_{0} @ 550 \mathrm{~nm}$ & \multicolumn{4}{|l|}{$0.19 \mathrm{~m}$} \\
\hline $\mathrm{r}_{0} @ 2200 \mathrm{~nm}$ & \multicolumn{4}{|l|}{$1.0 \mathrm{~m}$} \\
\hline $\mathrm{L}_{0}$ & \multicolumn{4}{|l|}{$22 \mathrm{~m}$} \\
\hline Layer altitude & $0 \mathrm{~m}$ & $4310 \mathrm{~m}$ & $10340 \mathrm{~m}$ & $16250 \mathrm{~m}$ \\
\hline Layer strength & 0.54 & 0.22 & 0.16 & 0.08 \\
\hline Wind speed & $7 \mathrm{~m} / \mathrm{s}$ & $30 \mathrm{~m} / \mathrm{s}$ & $20 \mathrm{~m} / \mathrm{s}$ & $8 \mathrm{~m} / \mathrm{s}$ \\
\hline
\end{tabular}

Table 1: Atmosphere parameters

We verified that our phase screens give the correct results in two cases: a $100 \mathrm{~m}$ and an $8 \mathrm{~m}$ pupil. The atmosphere evolved for 20 seconds with a time step of $20 \mathrm{~ms}$. We computed the wavefront variance and the Strehl ratio of the uncorrected long exposure image. An analytical approach [4] predicts the values reported in table.

\begin{tabular}{|l|l|l|l|l|}
\hline \multirow{2}{*}{ Pupil size } & \multicolumn{2}{|l|}{ Predicted values (2200nm) } & \multicolumn{2}{c|}{ Measured Values (2200 nm) } \\
\cline { 2 - 5 } & \multicolumn{1}{|c|}{ Strehl } & \multicolumn{1}{c|}{ Variance } & Strehl & Variance \\
\hline $8 \mathrm{~m}$ & $2.37 \%$ & $7.97 \mathrm{rad}^{2}$ & $2.93 \%$ & $5.85 \mathrm{rad}^{2}$ \\
\hline $100 \mathrm{~m}$ & $0.03 \%$ & $14.67 \mathrm{rad}^{2}$ & $0.02 \%$ & $18.44 \mathrm{rad}^{2}$ \\
\hline
\end{tabular}

Table 2: Comparison between predicted and simulated values for the wave front variance 


\subsection{Sensor and detector}

Our sensor is modeled as a phase sensor. The layer-oriented wavefront sensing architecture considered in this paper requires a pupil plane sensor like the pyramid one in order to take advantage from the co-adding of the photons coming from different guide stars to improve the signal to noise ratio. Given the similarities between the Shack-Hartmann and the pyramid wavefront sensor, we borrow the noise model from the well-known SH WFS described in [15] and we disturb the phase measurements with this model. We consider four different types of noise:

- Photon noise: $\sigma_{\gamma}^{2}=\frac{\pi^{2}}{2} \frac{1}{n_{p h}}\left(\frac{d}{r_{0 W F S}}\right)^{2}$, where $n_{p h}$ is the number of photons received in a single sub-aperture in one integration time.

- Read-out noise: $\sigma_{R O N}^{2}=\frac{\pi^{2}}{3} \frac{R O N^{2}}{n_{p h}^{2}}\left(\frac{2 d}{r_{O W F S}}\right)^{2} \cdot 4$, where RON is the read-out noise of one sub-aperture, which for the pyramid wave front sensor is composed by 4 pixels and for each pixel we consider a RON of $4 \mathrm{e}^{-}$.

- Dark: $\sigma_{D A R K}^{2}=\frac{\pi^{2}}{3} \frac{D A R K^{2}}{n_{p h}^{2}}\left(\frac{2 d}{r_{0 W F S}}\right)^{2} \cdot 4$, where the dark noise at $-60^{\circ}$ is about 10 electrons per second, so $0.01 \mathrm{e}^{-}$ per integration time. Compared to the RON, the dark noise is negligible.

- Sky: $\sigma_{S K Y}^{2}=\frac{4 \pi^{2}}{3} \frac{S K Y}{n_{p h}^{2}}\left(\frac{d}{r_{0 W F S}}\right)^{2}$, where SKY is the number of photons hitting each subaperture, considering a sky of $\mathrm{m}_{\mathrm{v}}=21$ per squared arcsec.

We don't consider the remaining background of the other stars in the field because designs exist that put the remaining light far from the detectors (central pupil with the background and the 4 pyramid pupils far enough, or reflective pyramids and the background absorbed). These 4 errors are added to determine the total variance of the phase error for each subaperture. Its standard deviation is used to model a Gaussian random variable and this error, converted in meters at the wave front sensing wavelength $(700 \mathrm{~nm})$, is added to the measured optical displacement.

\subsection{Guide star configuration}

We used the following relation for the photons received from a star of magnitude $0: \mathrm{V}_{0}=10^{11} \mathrm{ph} / \mathrm{m}^{2} / \mathrm{s} / \mu \mathrm{m}$. With a throughput of 0.2 , we have: $\mathrm{V}_{0}=2.10^{10} \mathrm{ph} / \mathrm{m}^{2} / \mathrm{s}$. We scale this number to the desired magnitude and telescope aperture. We define three types of stars:

- Guide stars inside the 2' FoV

- Guide stars outside the 2' Fov but inside the 6' FoV (1'-3' annular region)

- Sample stars, defined inside the 2' FoV

The choice of the stars of the first type is critical. Those stars must be selected in order to maximize the coverage of the upper layer DM. We choose to place those stars equally spaced on the circle of radius 1' around the centre, plus one additional star at the centre. The guide stars on the annular region can be chosen almost arbitrarily: we are interested in overlapping their light on the ground layer where they overlap perfectly no matter where they come from. We choose to have the guide stars on the circle of 3' radius around the centre.

The sample stars are virtual stars placed there only to measure the Strehl. Since the upper mirror will not be completely illuminated, there will be some pixels in this mirror that are not controlled. A more sophisticated algorithm that extrapolates the best values to apply to those pixels should be used (modal control), but for simplicity we did not implement it. The sample stars will hit, very likely, regions were the correction is not applied. To solve this problem we decided to mask the wave front seen by the sample star with the actual corrected area. We verified that the Strehl difference in the perfect case of a flat wave front is negligible, given also the large size of the pupil for the OWL case. The Strehl distribution is then computed using all the 2 ' FoV guide stars and the sample stars.

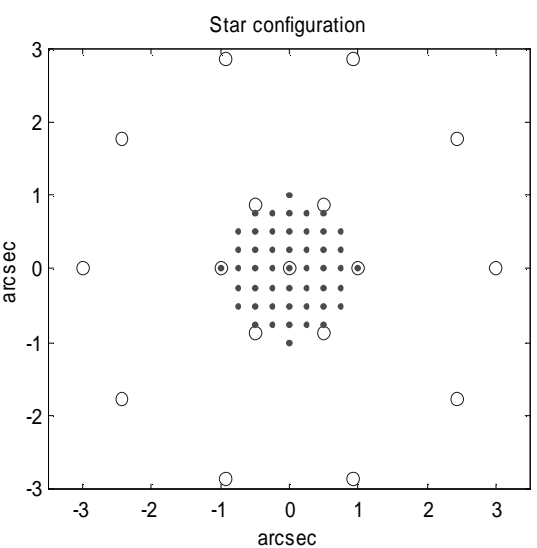

Figure 1: Guide and sample star configuration 
Another simplification is the fact that the light coming from the guide stars in the annular region does not pass through the upper layer DM. Although possible in principle, this is only a simulation shortcut and not a real requirement of the Multiple FoV architecture.

To speed-up the simulation, we do not compute the PSF except for the central star. Since for a long exposure time the centre of the PSF is located on the central pixel (verified on simulation), we compute directly the central point of the PSF without computing the 2D Fourier transform:

$$
F(\Omega, \Psi)=\sum_{m=-\infty}^{\infty} \sum_{n=-\infty}^{\infty} a[m, n] e^{j(\Omega m+\Psi n)} \Rightarrow F(0,0)=\sum_{m=-\infty}^{\infty} \sum_{n=-\infty}^{\infty} a[m, n]
$$

The value we are interested in is the squared modulus of the 2D FFT of the electrical field filtered by the telescope pupil computed in zero, so we can compute the Strehl ratio directly transforming the phase screens into the electrical field $f$ and dividing the result by the intensity of the image. The latter value can be computed using Parseval theorem:

$$
E=\sum \sum|f[m, n]|^{2}=\frac{1}{p^{2}} \sum \sum|F[m, n]|^{2}
$$

where $p^{2}$ is the number of pixels of the support of the DFT and the summation is extended to all the (masked) values in the $\operatorname{pxp}$ grid, so that the final result is:

$$
P S F_{\max }=\frac{\left|\sum_{m=-\infty}^{\infty} \sum_{n=-\infty}^{\infty} f[m, n]\right|^{2} p^{2}}{\sum \sum|f[m, n]|^{2}}
$$

We pre-computed the reference Strehl applying the same technique to a flat wave front and we related Equation (1) to that value.

\subsection{Mirrors}

The mirrors are implemented with a linear interpolation filter applied directly to the phase measurements disturbed by the noise.

Another shortcut of the simulation is the fact that every mirror has the same geometry as its conjugated sensor.

In this way the sensor measurements are directly applied to the mirror that has only to increase the resolution of the measures to match the resolution of the phase screens: this is done using linear interpolation. In Figure 2 we show a simple example that simulates a sensor with a 10x10 geometry and a phase screen with a 100x100 geometry with some actuator active: this technique models a mirror with pyramidal influence function.

The altitude of the mirrors is function of their geometry: this is another simplification of the simulation, that forces the phase screens resolution to

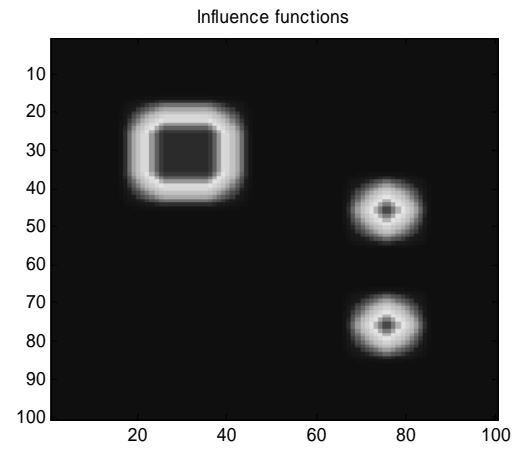

Figure 2: Influence functions be an integer multiple of the mirror/sensor geometry. The upper layer sensor and mirror are placed around $10 \mathrm{Km}$. Mirrors are controlled through an integrator with gain using directly the sensor measurements, skipping the computation of the interaction matrix. This is possible because the Layer-Oriented technique does not require reconstructing the volume of the turbulence, but works locally.

\section{SIMULATION ARCHITECTURE}

The large numbers involved like the size of the phase screens and the size of the pupil make an OWL simulation almost intractable with a single CPU and standard tools like Matlab and IDL. More CPUs are required.

We decided to use the experimental AO Beowulf cluster made of 6 PCs: the first PC, the master, is a dual processor system while the others have one processor each. As inter-processor communication library we used MPI. The simulation software has been developed entirely in $\mathrm{C}++$ and structured as a set of classes, as shown in Figure 4.

One time-consuming task for every MCAO simulation is the need to compute the beam propagation along

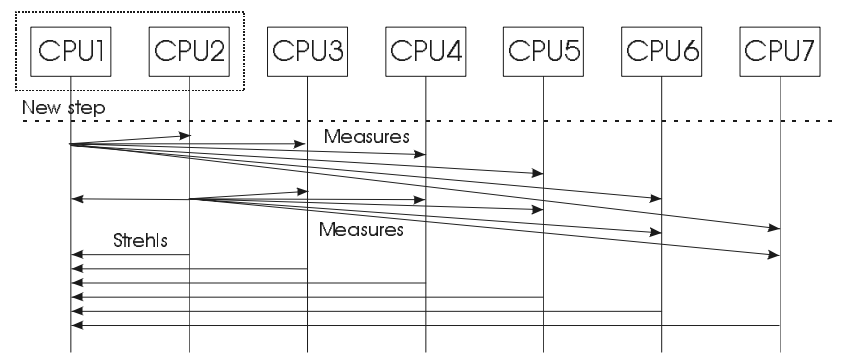

Figure 3: Sequence diagram for the OWL simulation 
several directions in order to evaluate the AO correction on the field of interest. We placed 69 sampling directions on a 9x9 grid.

We then dedicated CPUs 3 to 7 to the computation of the beam propagation along the sampling directions assigning an even number of directions to each CPU.

The first 2 CPUs, running on the same machine, instead compute the beam propagation of the guide stars and the simulation of the detectors. CPU1 computes the stars in the inner 2' FoV region and the two detectors there defined, while CPU2 computes the stars in the 1'-3' FoV annular region and the detector there defined. Every CPU runs the complete atmospheric model that evolves synchronously.

CPUs $1 \& 2$ accumulate the light coming from the various directions onto the detectors and then they produce the measures. Those measures are then broadcasted to all the other CPUs through MPI. Every CPU in the cluster knows the result of the wavefront measurements and each can compute the mirror controls. Meanwhile CPUs from 3 to 7 computed the beam propagation along all the sampling directions and the resulting Strehl. The Strehl is sent back to CPU1 from CPU2 for the stars in the annular region and from CPU3 to 7 for the sample stars. CPU1 collects all the results and compute the final integrated Strehl map for all the directions, along with its statistics. Figure 3 shows the sequence diagram we just described.

In the following paragraph we proceed testing the OWL simulation tool with some known configurations.

\section{VERIFICATIONS}

\subsection{Standard AO system, $8 \mathrm{~m}$ class}

We start our verification cases comparing our simulation with the results presented by Rigaut \& al. [14]. We set up our simulation to match Rigaut's conditions as much as possible.

\begin{tabular}{|l|l|l|}
\multicolumn{1}{|c|}{ Rigaut case } & \multicolumn{1}{l|}{ Our approximation } \\
\hline Telecope diameter & $7.9 \mathrm{~m}$ & $8.1 \mathrm{~m}$ \\
\hline Central obstruction & $1.2 \mathrm{~m}$ & $0 \mathrm{~m}$ \\
\hline $\mathrm{m}_{\mathrm{r}}=0$ photons/s & $8.2 \mathrm{e} 11$ & $8.2 \mathrm{e} 11$ \\
\hline Throughput & $50 \%$ & $50 \%$ \\
\hline Sky brightness & 20.3 & 20.3 \\
\hline CCD readout noise & $5 \mathrm{e}^{-}$ & $5 \mathrm{e}^{-}$ \\
\hline Wind speed & $20 \mathrm{~m} / \mathrm{s}$ & $20 \mathrm{~m} / \mathrm{s}$ \\
\hline Number of layers & 1 & 1 \\
\hline R0 at 550nm & $0.25\left(0.45^{\prime}\right)$ & $0.25\left(0.45^{\prime}\right)$ \\
\hline Wave-front sensor $\lambda$ & $700 \mathrm{~nm}$ & $700 \mathrm{~nm}$ \\
\hline Imaging $\lambda$ & $1600 \mathrm{~nm}$ & $1600 \mathrm{~nm}$ \\
\hline Pupil diameter (pixels) & 117 & 81 \\
\hline Sensor geometry & $9 \mathrm{x} 9$ & $9 \mathrm{x} 9$ \\
\hline
\end{tabular}

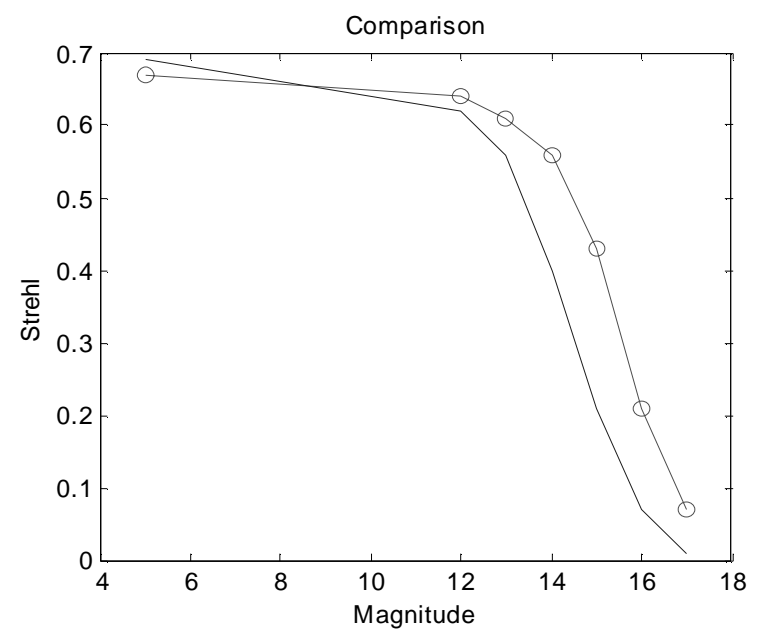

Figure 5: The curve with the circles is the reference case. The other curve is the one we obtain from our simulation which appears to be pessimistic compared to the Rigaut's case. Strehl given at $1600 \mathrm{~nm}$. 


\subsection{MCAO system, $8 \mathrm{~m}$ class}

The Multiconjugate Adaptive Optics Demonstrator (MAD) is a MCAO system under development at ESO and thus we have easy access to the design documents to compare the MAD forecasted performance with the results of our simulator. Layer-Oriented MAD uses two bimorph mirrors with 60 actuators each and two detectors with $8 \times 8$ and $7 x 7$ geometry, respectively ground and upper layer, single field-of-view (2'). We then configured our simulator with pupil with 80x80 pixels and the correct sensor geometry. The mirrors follow the geometry of the conjugated sensor and thus have 52 and 37 channels each.

We started first with a classical AO simulation, with only one mirror, one detector and one target at the center, without noise. The expected Strehl due only to fitting error is $67 \%$ and we found $71 \%$. We then simulated the cases indicated in the MAD System Analysis, which used a different atmosphere of 0.72 arcsec at 550nm and a different photon flux for $\mathrm{m}_{\mathrm{v}}=0$ equal to $5.37 \mathrm{e} 11 \mathrm{ph} / \mathrm{s}$. The number of stars in the 2 ' FoV is 8 .

\begin{tabular}{|c|c|c|c|c|}
\hline \multirow{2}{*}{$\begin{array}{l}\text { Integrated } \\
\text { magnitude }\end{array}$} & \multicolumn{2}{|c|}{ MAD Results } & \multicolumn{2}{|c|}{ Our Results } \\
\hline & Strehl $\pm \sigma$ & Max/PV & Strehl $\pm \sigma$ & Max/PV \\
\hline 9 & $22 \pm 5 \%$ & $35 \% / 25 \%$ & $25 \pm 2 \%$ & $29 \% / 8 \%$ \\
\hline 10 & $21 \pm 5 \%$ & $33 \% / 23 \%$ & $24 \pm 2 \%$ & $28 \% / 8 \%$ \\
\hline 11 & $18 \pm 4 \%$ & $28 \% / 20 \%$ & $20 \pm 2 \%$ & $24 \% / 7 \%$ \\
\hline 12 & $10 \pm 2 \%$ & $15 \% / 11 \%$ & $11 \pm 1 \%$ & $14 \% / 5 \%$ \\
\hline 13 & $3 \pm 1 \%$ & $4 \% / 2 \%$ & $2 \pm 1 \%$ & $2 \% / 1 \%$ \\
\hline
\end{tabular}

Table 3: Comparison with MAD analysis

Results are well aligned even if our simulation delivers a better uniformity in the correction. A possible explanation is that in our case we used only 4 layers to simulate the atmosphere while original MAD simulations used the complete Cerro-Pachon model with 7 layers.

\subsection{MCAO system, $100 \mathrm{~m}$ class}

We now move to the $100 \mathrm{~m}$ class telescope, the OWL case. Using a sensor geometry of 100x100 and the corresponding mirror geometry of 100x100 we have 7869 for the number of active subapertures. The expected Strehl due only to fitting error is $67 \%$ and we found $70 \%$. We then verified that the anisoplanatism behaves as expected. In our conditions $\theta_{0}$ is equal to:

$$
\theta_{0}=0.314 \frac{r_{0}}{\bar{h}}=0.314 \frac{1}{3902}=16.6 "
$$

We ran a simulation with OWL in a classical AO configuration with a single DM and one guide star at the center and we compared the resulting Strehl on one axis of the 2' FoV with the effect of the anisoplanatism. Figure 6 shows that the agreement is very good.

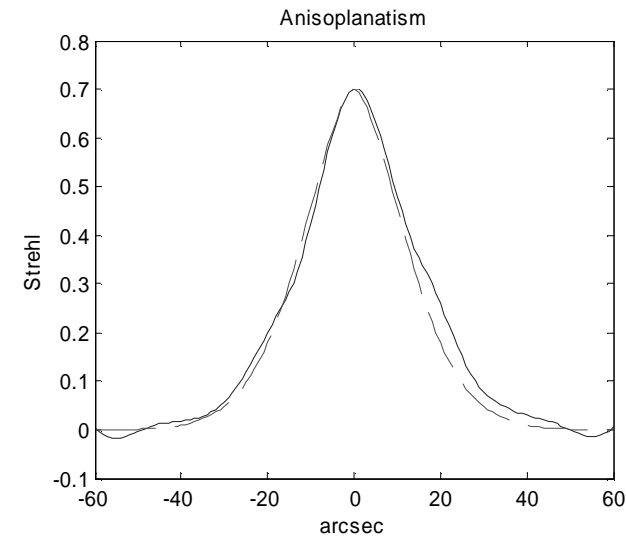

Figure 6: Anisoplanatism

\section{MAD-OWL COMPARISON}

We are now ready to run our first complete simulations. We start with few simple cases comparing the MAD configuration with the OWL one using the same base parameters (seeing and reference flux). For both we used 7 very bright guide stars in the 2' field of view to determine the performance upper limit. The last case considers the Multiple Field-of-View technique for both configurations and we added 10 stars in the annular region between 1' and 3' radius. We did not perform any optimization to decide which beam splitter or which controller to use. We used the MAD configuration that will be used in reality on the MAD

\begin{tabular}{|l|l|l|l|}
\hline \multicolumn{2}{|c|}{ MAD } & OWL \\
\hline \multicolumn{2}{|l|}{ Telescope pupil } & $8 \mathrm{~m}$ & $100 \mathrm{~m}$ \\
\hline R0 @ 2200 nm & $1.0 \mathrm{~m}$ & $1.0 \mathrm{~m}$ \\
\hline \hline \multirow{2}{*}{$\begin{array}{l}\text { Ground } \\
\text { layer }\end{array}$} & Sampling & $8 \times 8$ & $100 \times 100$ \\
\cline { 2 - 4 } & Sub-aperture size & $1.0 \mathrm{~m}$ & $1.0 \mathrm{~m}$ \\
\hline \hline \multirow{4}{*}{$\begin{array}{l}\text { layer } \\
\text { layer }\end{array}$} & Altitude & $10320 \mathrm{~m}$ & $10320 \mathrm{~m}$ \\
\cline { 2 - 4 } & Meta-pupil size & $14 \mathrm{~m}$ & $105.3 \mathrm{~m}$ \\
\cline { 2 - 4 } & Sampling & $7 \times 7$ & $53 \times 53$ \\
\cline { 2 - 4 } & Sub-aperture size & $2.0 \mathrm{~m}$ & $2.0 \mathrm{~m}$ \\
\hline
\end{tabular}

Table 4: Simulation configuration 
project: the ground layer sensor uses an array of $8 \times 8$ sub-apertures, while the upper layer uses a grid of $7 \times 7$ subapertures. Our simulated atmosphere defines an $\mathrm{r} 0=1.0 \mathrm{~m}$ at $2200 \mathrm{~nm}$, our imaging wavelength. We then define an equivalent OWL configuration to set up the simulation.

\begin{tabular}{|c|l|c|c|}
\hline$\#$ & Case & \multicolumn{1}{|c|}{$\begin{array}{c}\text { MAD } \\
\text { Peak, <Strehl } \pm \sigma\end{array}$} & $\begin{array}{c}\text { OWL } \\
\text { Peak }\end{array}$ \\
\hline 1 & Only ground DM active, one guide star at the center, gain =0.9 $\pm \sigma$ \\
\hline 2 & Only ground DM, 7 guide stars on 2' FoV, 1 at the centre, gain =0.9 & $70 \%, 11 \pm 15 \%$ & $70 \%, 9 \pm 15 \%$ \\
\hline 3 & $\begin{array}{l}\text { Ground DM and Upper DM active, 7 guide stars on 2' FoV, 1 of them } \\
\text { at the centre, gain =0.8/0.8. Single FoV Layer Oriented }\end{array}$ & $42 \%, 32 \pm 4 \%$ & $43,38 \pm 3 \%$ \\
\hline 4 & $\begin{array}{l}\text { Ground DM and Upper DM active, 7 guide stars on 2' FoV, 1 of them } \\
\text { at the centre and those stars are used only to drive the upper DM, 10 } \\
\text { guide stars on 2'-6' FoV annulus and those are used to drive the } \\
\text { ground DM, gain =0.8/0.8. Single FoV Layer Oriented }\end{array}$ & $35 \%, 26 \pm 4 \%$ & $28 \%, 19 \pm 4 \%$ \\
\hline 5 & $\begin{array}{l}\text { Full Multiple Field-of-View MCAO, 7 guide stars on 2' plus 7 guide } \\
\text { stars on the annulus 2'-6'. Gains: 0.4/0.8 (ground/upper) and 0.8 for } \\
\text { the third loop. Beam splitter 50\%/50\% for the 2' FoV. }\end{array}$ & $40 \%, 33 \pm 3 \%$ & $42 \%, 32 \pm 5 \%$ \\
\hline
\end{tabular}

\subsection{MAD Optimizations for the Multiple Field-of-View case}

Since the performance of the two systems are quite similar, we decided to explore the parameter space for MAD since one full MAD simulation completes much quicker than an OWL one. We will then use the optimized parameters to set up the OWL simulations. We start from the same configuration as in the last of the previous simulations, with 7 stars in the 2 ' FoV and 10 on the annular region. Beam splitter and controller configurations are identical.

\subsubsection{Integration time optimization}

We run a set of simulations changing the magnitude of the guide stars, which are all identical. This will proceed towards the faint regime to take into account the effect of the noise. Our aim is to find which integration time gives the best performance. The three numbers on the first column are as follows: magnitude of each of the $7+10$ stars, integrated magnitude of the 7 stars on the 2' field, integrated magnitude of the 10 stars on the 1'-3' annular region, Strehl values are mean and rms on the 2' FoV.

\begin{tabular}{|l|l|l|l|l|l|l|l|}
\hline Magnitude & $\mathbf{1 m s}$ & $\mathbf{2 m s}$ & $\mathbf{5 m s}$ & $\mathbf{1 0 m s}$ & $\mathbf{2 0 m s}$ & $\mathbf{5 0 m s}$ & $\mathbf{1 0 0 m s}$ \\
\hline $10(7.8 / 7.5)$ & $\mathbf{3 3} \pm \mathbf{3 \%}$ & $33 \pm 3 \%$ & $32 \pm 2 \%$ & $30 \pm 2 \%$ & - & - & - \\
\hline $12(9.8 / 9.5)$ & $\mathbf{3 3} \pm \mathbf{3 \%}$ & $33 \pm 3 \%$ & $33 \pm 3 \%$ & $30 \pm 2 \%$ & - & - & - \\
\hline $14(11.8 / 11.5)$ & $30 \pm 2 \%$ & $\mathbf{3 2} \pm \mathbf{3 \%}$ & $31 \pm 3 \%$ & $30 \pm 1 \%$ & - & - & - \\
\hline $16(13.8 / 13.5)$ & $9 \pm 2 \%$ & $20 \pm 3 \%$ & $28 \pm 3 \%$ & $\mathbf{2 9} \pm \mathbf{2 \%}$ & $23 \pm 2 \%$ & - & - \\
\hline $18(15.8 / 15.5)$ & - & - & $5 \pm 1 \%$ & $14 \pm 2 \%$ & $\mathbf{1 7} \pm \mathbf{2 \%}$ & $10 \pm 1 \%$ & - \\
\hline $20(17.8 / 17.5)$ & - & - & - & - & $1 \pm 0.5 \%$ & $\mathbf{4} \pm \mathbf{1 \%}$ & $4 \pm 1 \%$ \\
\hline
\end{tabular}

\subsubsection{Gain/Beam splitter optimization:}

We selected one case from the previous table and we tried to optimize the beam splitter and the controller. The light coming from the central 2' has to be shared between the two detectors collecting it, one conjugated to the ground and one to the upper layer. While in Single FoV mode the choice 50\%/50\% seems to be reasonable, it is not at all obvious for the Multiple FoV what is the best combination since the ground layer is sampled also using the light coming from the 1'-3' annular region.

We ran several simulations with different parameters but we did not find a recipe by which control parameters can be chosen and the variation in performance was minimal. This optimisation seems to play a little role.

\subsubsection{Wavefront Sampling optimization:}

The simple structure of the simulation software does not allow us to span a wide set of sensor configurations. For the MAD case we can test only two configurations: the default one and one with double resolution. With OWL we have more freedom, but of course each OWL simulation cost significantly more than the MAD ones making more difficult the process of optimization of the wave front sampling. 


\begin{tabular}{|c|c|c|}
\hline Magnitude & $\begin{array}{c}\text { 8x8/7x7 (coarse) } \\
<\text { Strehl }> \pm \sigma\end{array}$ & $\begin{array}{c}\text { 16x16/14x14 (fine) } \\
<\text { Strehl }> \pm \sigma\end{array}$ \\
\hline $10(7.8 / 7.5)$ & $32 \pm 3 \%$ & $41 \pm 5 \%$ \\
\hline $14(11.8 / 11.5)$ & $31 \pm 3 \%$ & $35 \pm 4 \%$ \\
\hline $18(15.8 / 15.5)$ & $18 \pm 2 \%$ & Very low \\
\hline
\end{tabular}

We observe that magnitude 14 seems to be the threshold above which the finer resolution is convenient. We also observe that the optimized parameters for the controller did not improve performance for magnitude 14 and 10 .

\subsection{Simulations cases for MAD Multiple Field-of-View}

We then defined three interesting cases to simulate with OWL, using as the baseline the cases identified by Marchetti in [6]. These are the cases:

\begin{tabular}{|l|l|l|l|}
\hline Galactic Latitude: & $\mathbf{2 0}$ & $\mathbf{5 0}$ & $\mathbf{9 0}$ \\
\hline Number of stars in 2' FoV & 6 & 5 & 4 \\
\hline Integrated magnitude in 2' FoV & 14 & 16 & 16 \\
\hline Probability of this star configuration & $92 \%$ & $33 \%$ & $15 \%$ \\
\hline Number of stars in 1'-3' annular region & 8 & 5 & 5 \\
\hline Integrated magnitude in 1'-3' annular region & 12 & 12 & 13 \\
\hline Probability of this star configuration & $93 \%$ & $89 \%$ & $80 \%$ \\
\hline Combined probability & $89 \%$ & $29 \%$ & $12 \%$ \\
\hline
\end{tabular}

Simulating those cases with MAD we obtain (values in the table are mean and rms Strehl on 2' FoV):

\begin{tabular}{|l|l|l|l|l|l|l|l|}
\hline \multirow{2}{*}{ Latitude } & $\mathbf{1 m s}$ & $\mathbf{2 m s}$ & $\mathbf{5 m s}$ & $\mathbf{1 0 m s}$ & $\mathbf{2 0 m s}$ & $\mathbf{5 0 m s}$ & Sampling \\
\hline \multirow{2}{*}{50} & - & $21 \pm 2 \%$ & $27 \pm 2 \%$ & $27 \pm 2 \%$ & $22 \pm 3 \%$ & - & Coarse \\
\cline { 2 - 8 } & - & $11 \pm 2 \%$ & $29 \pm 3 \%$ & $\mathbf{3 2 \pm 3 \%}$ & $27 \pm 3 \%$ & - & Fine \\
\hline \multirow{3}{*}{50} & - & - & $5 \pm 1 \%$ & $14 \pm 2 \%$ & $\mathbf{1 6} \pm \mathbf{2 \%}$ & $10 \pm 2 \%$ & Coarse \\
\cline { 2 - 8 } & - & - & - & $5 \pm 1 \%$ & $13 \pm 3 \%$ & $10 \pm 2 \%$ & Fine \\
\hline \multirow{2}{*}{90} & - & - & $3 \pm 1 \%$ & $11 \pm 3 \%$ & $\mathbf{1 4 \pm 2 \%}$ & $10 \pm 2 \%$ & Coarse \\
\cline { 2 - 8 } & - & - & - & - & - & - & Fine \\
\hline
\end{tabular}

\section{RESULTS}

In the previous sections we searched the parameter space using the MAD configuration. We have now defined three simulation cases for the three coordinates of interest along with the loop parameters. We will run two complete sets of simulations for OWL, one in Single Field-of-View mode and the other in Dual Field-of-View mode with a number of guide stars and integrated magnitude according to the table, but placed symmetrically like in the previous sections.

Additionally, we will run a simulation on a real configuration, simulating a telescope pointing at the South Galactic Pole configuring the asterism with the real star distribution that is present there.

In this section the sky coverage is defined as the probability to find an asterism with the given configuration of number of stars and integrated magnitude in the given sky region.

\subsection{Performance for OWL in Single Field-of-View mode}

We summarize the results for the first set of OWL simulations in the following table

\begin{tabular}{|l|l|l|l|}
\hline Latitude & 20 & 50 & 90 \\
\hline Magnitude per star in 2' FoV & 15.9 & 17.7 & 17.5 \\
\hline Strehl & $\mathbf{3 7 \pm 3 \%}$ & $\mathbf{1 7 \pm 2} \%$ & $\mathbf{1 6} \pm \mathbf{3} \%$ \\
\hline Max Strehl / Peak-to-valley & $43 \% / 14 \%$ & $21 \% / 8 \%$ & $21 \% / 12 \%$ \\
\hline Exposure time & $4 \mathrm{~s}$ & $4 \mathrm{~s}$ & $4 \mathrm{~s}$ \\
\hline Integration time & $10 \mathrm{~ms}$ & $20 \mathrm{~ms}$ & $20 \mathrm{~ms}$ \\
\hline Geometry & Fine & Coarse & Coarse \\
\hline Sky coverage & $92 \%$ & $33 \%$ & $15 \%$ \\
\hline
\end{tabular}




\subsection{Performance for OWL in Multiple Field-of-View mode}

\section{Figure 7: Strehl Map}

Simulation parameters:

- Coordinates: galactic plane $\left(20^{\circ} \mathrm{LAT}\right)$

- Stars in 2' FoV: 6;

- Integrated magnitude: 14 ;

- Equally distributed: 15.9 per star;

- Probability: $92 \%$

- Stars in 2'-6' FoV: 8;

- Integrated magnitude: 12 ;

- Equally distributed: 14.3 per star;

- Probability: $93 \%$

- $4 \mathrm{~s}$ exposure, $10 \mathrm{~ms}$ integration time

Results: 36 $\pm 4 \%$ Strehl on 2' FoV

Max: $46 \%$ Strehl, PV: $18 \%$

Sky coverage: $89 \%$ on galactic plane

\section{Figure 8: Strehl Map}

Simulation parameters:

- Coordinates: intermediate latitude $\left(50^{\circ} \mathrm{LAT}\right)$

- Stars in 2' FoV: 5;

- Integrated magnitude: 16;

- Equally distributed: 17.7 per star;

- Probability: $33 \%$

- Stars in 2'-6' FoV: 5;

- Integrated magnitude: 12 ,

- Equally distributed: 13.7 per star;

- Probability: $89 \%$

- 4s exposure, $20 \mathrm{~ms}$ integration time

Results: 15 $\pm 3 \%$ Strehl on 2' FoV

Max: 21\% Strehl, PV: $11 \%$

Sky coverage: $29 \%$ on intermediate latitude

\section{Figure 9: Strehl Map}

Simulation parameters:

- Coordinates: galactic pole $\left(90^{\circ} \mathrm{LAT}\right)$

- Stars in 2' FoV: 4;

- Integrated magnitude: 16;

- Equally distributed: 17.5 per star;

- Probability: $15 \%$

- Stars in 2'-6' FoV: 5;

- Integrated magnitude: 13 ,

- Equally distributed: 14.7 per star;

- Probability: $80 \%$

- $4 \mathrm{~s}$ exposure, $20 \mathrm{~ms}$ integration time Results: $15 \pm 4 \%$ Strehl on 2' FoV

Max: 21\% Strehl, PV: $13 \%$

Sky coverage: $12 \%$ on galactic pole
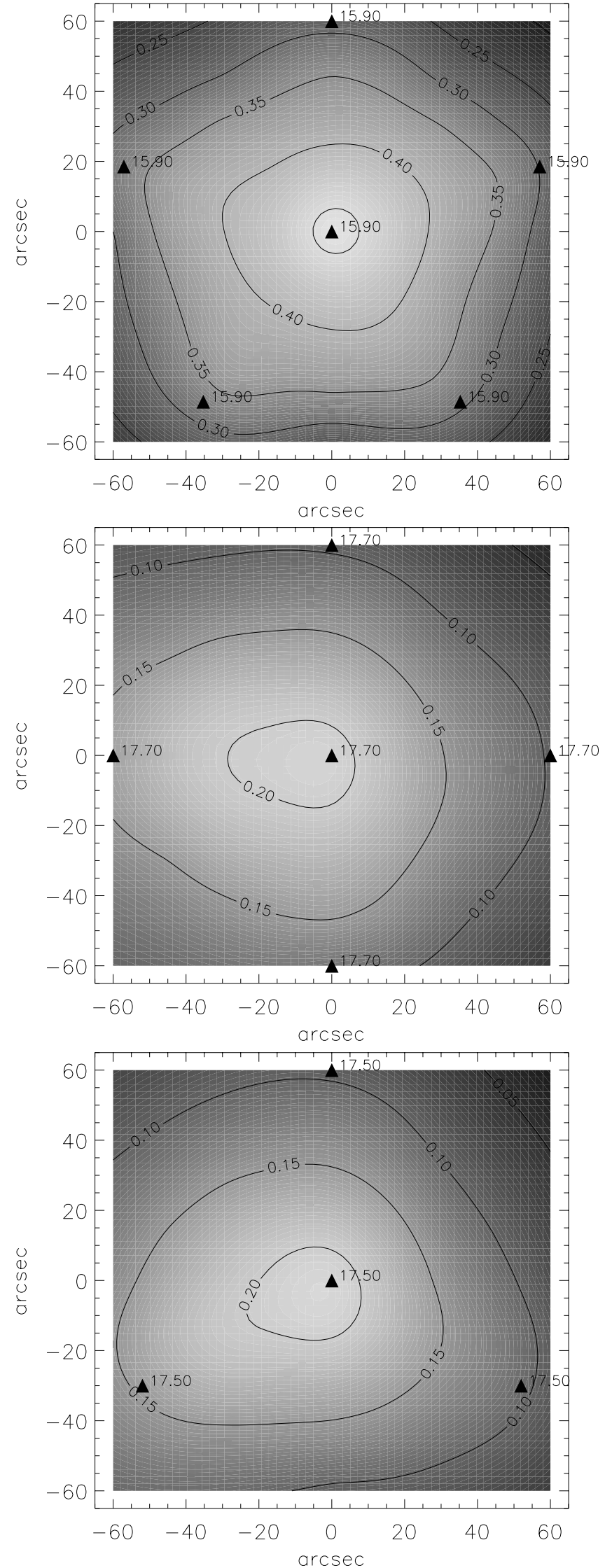


\subsection{Performance for OWL in a real case in Multiple Field-of-View mode}

We tried to simulate a real case. To make it more interesting we chose a coordinate close to the South Galactic Pole and few stars around it. The reason for this choice is that the SGP is a potential target for MAD and thus a good comparison case. Figure 12 shows the chosen geometry of the guide stars along with their magnitude (a bigger circle means a brighter star); all the stars in the field are used for correction. Figure 14 shows a picture of the area taken from the ESO catalogue while Figure 4 shows the final Strehl map with an exposure of 5 s Simulation parameters are hereby briefly reported. We also run this configuration with OWL in Single Field-of-View mode and MAD in both Single and Dual FoV for comparison.

\section{Figure 10: Strehl Map}

Simulation parameters:

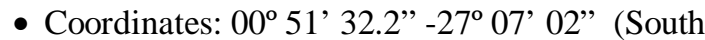
Galactic Pole)

- Stars in 2' FoV: 5; Integrated magnitude: 16.13;

- Magnitude distribution: $17.9 \pm 0.3$

- Stars in 2'-6' FoV: 11; Integrated magnitude: 14.97 ,

- Magnitude distribution: $17.7 \pm 0.5$

- Exposure time: $5 \mathrm{~s}$

- Integration time: $20 \mathrm{~ms}$

- Beam splitter: $30 \%, 70 \%$ on 2'

Results: $14 \pm 3 \%$ Strehl on 2' FoV Max: 18\% Strehl, PV: $11 \%$

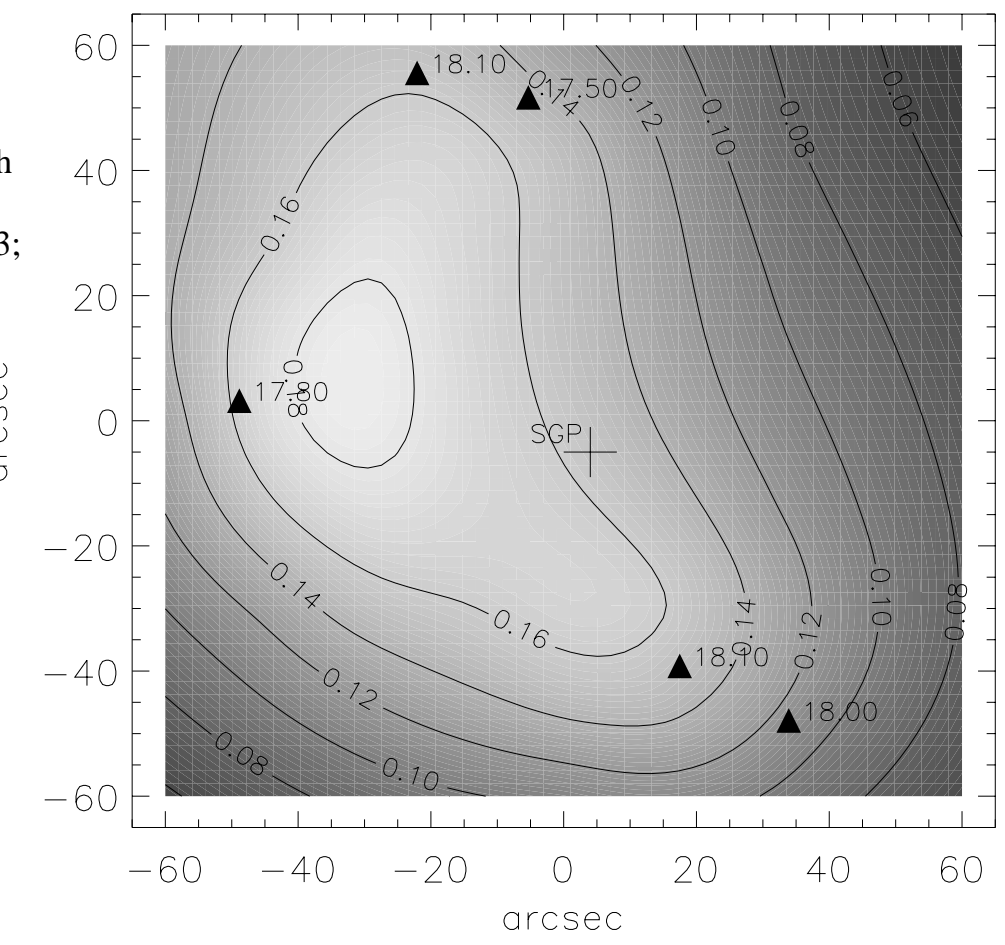

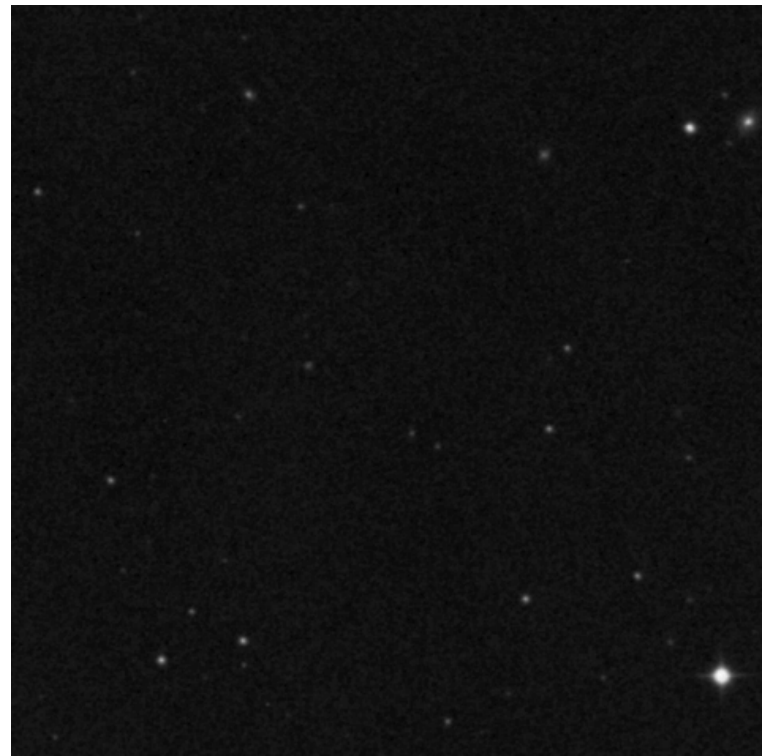

Figure 14: Picture of the sky are considered (6')

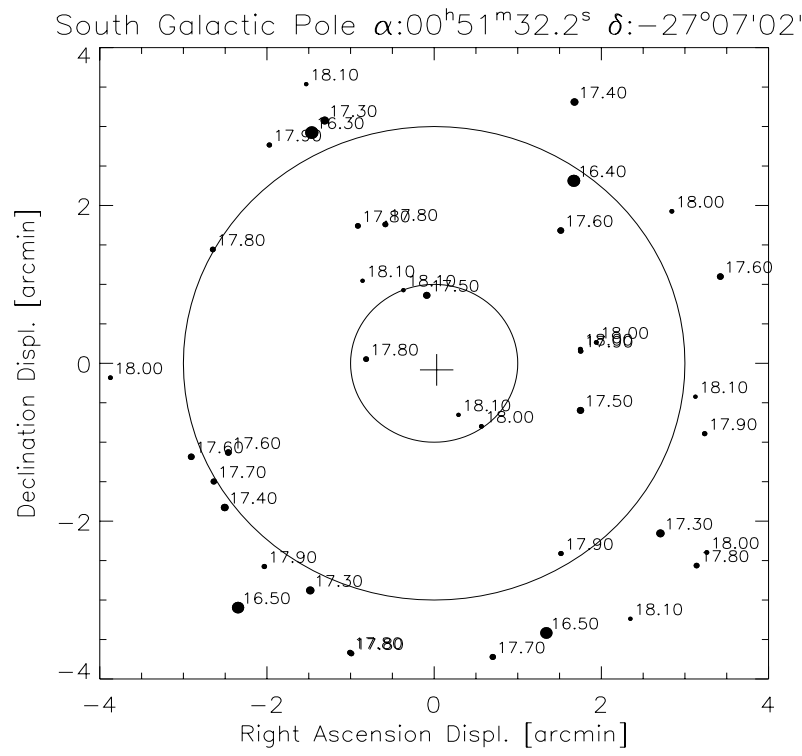

Figure 12: Star distribution on the field (8') 
We ran also the same configuration with OWL in Single FoV mode and MAD in both cases. The following table summarizes the results.

\begin{tabular}{|c|c|c|}
\hline Case & Performance & Peak/PV \\
\hline OWL M-FoV & $14 \pm 3 \%$ & $18 \% / 11 \%$ \\
\hline OWL S-FoV & $13 \pm 4 \%$ & $20 \% / 13 \%$ \\
\hline MAD M-FoV & $13 \pm 3 \%$ & $21 \% / 14 \%$ \\
\hline MAD S-FoV & $8 \pm 3 \%$ & $16 \% / 12 \%$ \\
\hline
\end{tabular}

Table 5: Summary of the results for the South Galactic Pole

The figure on the right is the Strehl map for the S-FoV OWL case.

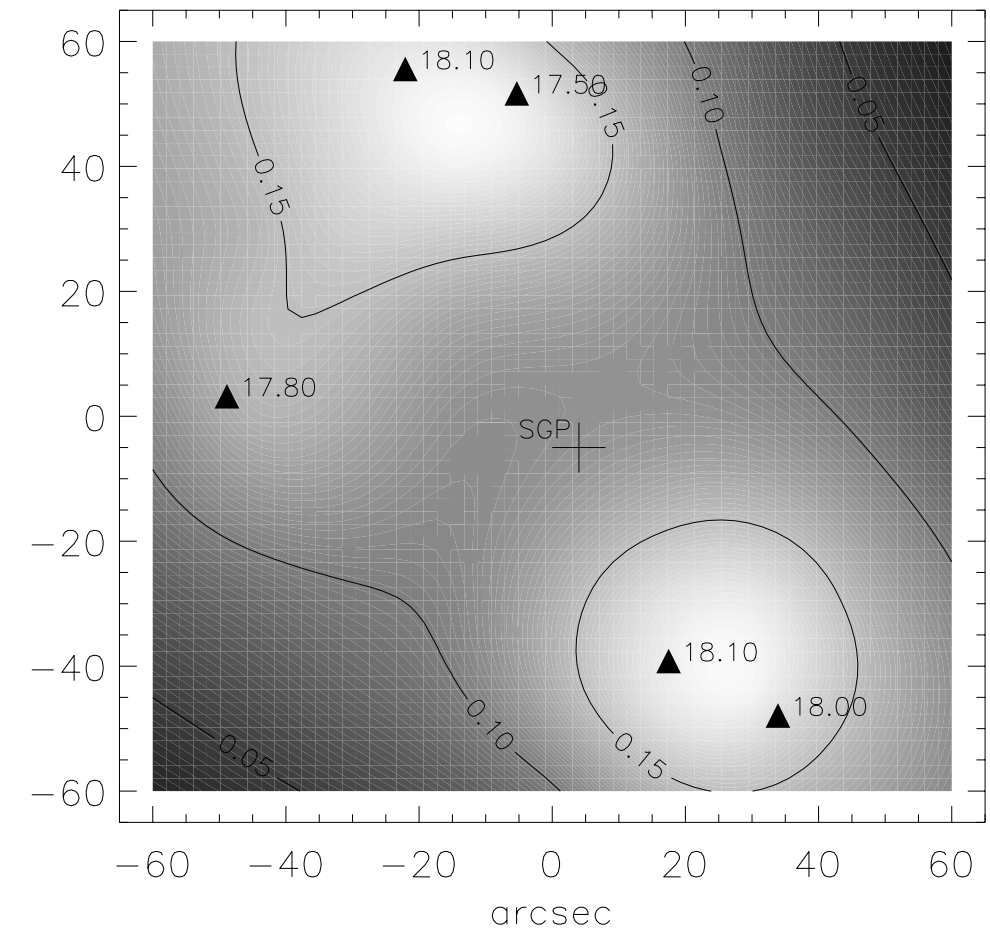

\subsection{The faint end}

We finally investigated how the Dual Field-of-View architecture behaves in comparison with the Single Field-of-View architecture. The previous simulations show that on the bright end it is possible to optimize the two systems in order to achieve similar performance. We then selected the two fainter cases (intermediate latitude and galactic pole) and we ran two more simulations for each case using stars 1 and 2 magnitudes fainter. Table 6 summarizes the results.

The case at $50^{\circ}$ uses 5 guide stars on the $2^{\prime}$ FoV and 5 stars on the annulus 2' $-6^{\prime}$ '. The case $90^{\circ}$ uses 4 guide stars on 2 ' FoV and 5 stars on the annulus 2' -6 ', identical to the condition of the previous simulations that are also reported here in bold for comparison. One clearly sees that the Dual Field-of-View architecture performs better than the Single Field-of-View architecture on the faint regime.

\begin{tabular}{|l|l|l|l|l|}
\hline \multirow{2}{*}{ Case } & \multicolumn{2}{|c|}{$\begin{array}{c}\text { Integrated } \\
\text { Magnitude }\end{array}$} & \multirow{2}{*}{ Single FoV } & Dual FoV \\
\cline { 2 - 3 } & $\mathbf{2}$ & $\mathbf{2}-\mathbf{6}^{\prime}$ & & \\
\hline $\mathbf{5 0}$ & $\mathbf{1 6}$ & $\mathbf{1 2}$ & $\mathbf{1 7} \pm \mathbf{2} \%$ & $\mathbf{1 6} \pm \mathbf{3} \%$ \\
\hline 50 & 17 & 13 & $7 \pm 1 \%$ & $10 \pm 2 \%$ \\
\hline 50 & 18 & 14 & Very low & $1 \pm 0.5 \%$ \\
\hline $\mathbf{9 0}$ & $\mathbf{1 6}$ & $\mathbf{1 3}$ & $\mathbf{1 6} \pm \mathbf{3 \%}$ & $\mathbf{1 6} \pm \mathbf{4} \%$ \\
\hline 90 & 17 & 14 & $6 \pm 1 \%$ & $9 \pm 2 \%$ \\
\hline 90 & 18 & 15 & Very low & $1 \pm 0.5 \%$ \\
\hline
\end{tabular}

Table 6: Simulation results

\section{CONCLUSIONS}

In this paper we presented a preliminary set of simulations for a $100 \mathrm{~m}$ class telescope like OWL. We solved the problems that a simulation of this size causes taking several shortcuts to simplify the computation. Even if simplified, we proved that the simulation tool delivers a good approximation of the performance of known systems. For the first time we presented an estimation for the performance a telescope like OWL can deliver using both Single Field-of-View and Dual Field-of-View Layer-Oriented technique.

In particular we verified that the M-FoV technique delivers a better mean Strehl ratio in the FoV of interest when compared with the S-FoV results. As expected, M-FoV also reduces the variance of the Strehl in the FoV of interest resulting in a better correction in the whole field.

Layer-Oriented (Single or Dual) applied to OWL benefits from the better pupil overlap at the high altitude layer resulting in better final performance. 
These preliminary results are encouraging since they show the layer-oriented architecture working in extreme cases like the South Galactic Pole and leave room for improving the performance implementing more advanced techniques like waffle mode control and modal control, which were not tackled in this paper.

We also proved that a computer cluster is well suited to run simulations of this size. As far as the software is concerned, the next step is to remove all the constraints we adopted and move toward a complete MCAO simulation tool.

We also identified an interesting topic of investigation comparing the Single FoV and the Multiple FoV technique: at a bright regime both techniques deliver the same performance, but in the faint regime the Multiple FoV performs better.

\section{ACKNOWLEDGEMENTS}

We would like to thank the ESO IT Department and in particular Giorgio Filippi who provided us with the majority of the computers composing the Beowulf cluster and supported us with his department; Stefano Turolla (ESO-IT) for his help in the cluster installation; Miska Le Louarn (ESO-AO) and Rodolphe Conan (RTN-AO) for their collaboration on the experiments with the Beowulf cluster and MPI.

The authors wish to acknowledge that this research is supported by the European Commission RTC program: "Adaptive Optics for Extremely Large Telescopes”, under contract \#HPRN-CT-2000-00147.

\section{REFERENCES}

1. Beckers J. M., "Increasing the size of the isoplanatic patch size with multiconjugate adaptive optics", in ESO conference on Very Large Telescopes and their instrumentation, M.-H. Hulrich, ed., pp. 693, 1988.

2. Beckers J. M., "Detailed compensation of atmospheric seeing using multiconjugate adaptive optics", Proc. SPIE 1114, pp. 215-217, 1989.

3. Beckers J. M., “Adaptive optics for astronomy -- Principles, performance, and applications”, ARA\&A 31, pp. 13-62, 1993.

4. Conan R, "Modélisation des effets de l'échelle externe de cohérence spatiale du front d'onde pour l'observation à Haute Résolution Angulaire en Astronomie", Ph.D. thesis, Université de Nice - Sophia Antipolis, 2000

5. Gilmozzi R., Delabre B., Dierickx P., Hubin N., Koch F., Monnet G., Quattri M., Rigaut F., Wilson R.N., "Future of filled aperture telescopes: is a 100-m feasible?", Proc. SPIE 3352, pp. 778-791, 1998.

6. Marchetti E, Ragazzoni R and Diolaiti E, "Which range of magnitudes for Layer Oriented MCAO?", SPIE 2002 submitted.

7. Ragazzoni R., "Pupil plane wave front sensing with an oscillating prism", J. of Mod. Opt. 43, pp. 289-293, 1996.

8. Ragazzoni R., Marchetti E. and Rigaut F., "Modal tomography for adaptive optics", A\&A 342, pp. L53-L56, 1999.

9. Ragazzoni R., "Adaptive optics for giants telescopes: NGS vs. LGS", in ESO Proocedings of the Bäckaskog Workshop on Extermely large Telescopes 57, T. Andersen, A. Ardeberg and R. Gilmozzi, eds., pp. 175-180, 2000.

10. Ragazzoni R., Marchetti E. and Valente G., "Adaptive-optics correction available for the whole sky", Nature 403, pp. 54-56, 2000.

11. Ragazzoni R., Farinato J. and Marchetti E., “Adaptive optics for 100-m-class telescopes: new challenges require new solutions", Proc. SPIE 4007, pp. 1076-1087, 2000.

12. Ragazzoni R., Diolaiti E., Farinato J., Fedrigo E., Marchetti E., Tordi M. and Kirkman D., "Multiple Field of View layer oriented", in ESO proceedings of the Venice conference on Beyond Conventional Adaptive Optics, R. Ragazzoni, N. Hubin and S. Esposito, eds., in publication., 2001a.

13. Ragazzoni R., Diolaiti E., Farinato J., Fedrigo E., Marchetti E., Tordi M. and Kirkman D., "Multiple Field of View layer oriented adaptive optics", A\&A submitted, $2001 \mathrm{~b}$.

14. Rigaut F., Ellerbroek L. and Northcott M., "Comparison of curvature based and Shack-Hartmann-based adaptive optics for the Gemini telescope", Applied Optics, pp 2856-2867, 1997

15. Rousset G, Primot J and Fontanella J-C., "Visible wavefront sensor development", in Workshop on Adaptive Optics in Solar Observations, Lest Foundation Technical Report 28, pp. 17-34, University of Oslo, Oslo.

16. Tallon M. and Foy R., "Adaptive telescope with laser probe - Isoplanatism and cone effect”, A\&A 235, pp. 549$557,1990$. 